\title{
THE ROLE OF UNIVERSITIES IN SUPPORTING THE UNITED STATES EFFORT TO COMBAT BIOTERRORISM FROM A NON-TRADITIONAL PERSPECTIVE
}

\author{
Michael Meagher \\ Professor of Chemical Engineering \\ Director, Biological Process Development Facility \\ University of Nebraska - Lincoln
}

Challenges Facing the National Institutes of Health and the U.S. Government

The U.S. government must protect the civilian population against biological agents that under "normal" circumstances have been significantly reduced or eliminated, such as anthrax and small pox. The challenge is to develop countermeasures to combat biological agents. The Department of Defense, in particular the U.S. Army Medical Research Institute of Infectious Diseases (USAMRIID), has been developing countermeasures against bioterrorism agents for the last 30 years. The Army's efforts concern only the solider in combat situations, which has an impact on how the Army addresses the issue of developing countermeasures. For example, the Army is only concerned about treating a select group of combat soldiers - young healthy adults with no known health problems. Also, the Army may only have to treat at most 200,000 to 300,000 soldiers. The Army's demographics do not completely fit the civilian population, where the most severely affected individuals from a bioterrorism attack will be the young and old, immuno-compromised, and the sick. The good news is that the technology developed by USAMRIID has applications for the civilian population, but production capabilities will need to increase nearly 100 fold and clinical testing will need to include a wider spectrum of society.

The next challenge is that a majority of the vaccines or therapeutic molecules against biological agents are of little monetary value to the pharmaceutical and biopharmaceutical industry. As an example, the Department of Defense (DoD) and the National Institute of Allergy and Infectious Diseases (NIAID) would like to have monoclonal antibodies (MAb) against biological agents to treat exposed individuals that are not vaccinated or cannot be vaccinated, i.e. vaccinating against botulism eliminates the therapeutic potential of Botox. At present, the biopharmaceutical industry pipeline is so full that the industry lacks the resources, especially production capacity, to produce the drugs that are already approved for the market and those in clinical trials. Enbrel is a MAb for treatment of juvenile rheumatoid arthritis and is produced by Immunex. Presently, Immunex has only enough production capacity to meet approximately $60 \%$ to $70 \%$ of the market needs. Immunex is building additional production facilities, but this will take three to five years to complete. At present the biopharmaceutical industry is challenged to find facilities to produce material for 
clinical studies, especially smaller companies who lack the resources to build and validate cGMP facilities.

On the other side are the contract manufacturing organizations (CMO) that are designed to meet the needs of the biopharmaceutical industry. Because of the significant investment to build and validate a commercial facility, the CMO are primarily interested in companies with advanced product portfolios that have multiple products and can commit to a long term relationship (five years or more). The goal of each $\mathrm{CMO}$ is to be the primary manufacturer of a fully approved product. The major CMOs, such as DSM and Diosynth, and large biopharmaceutical companies that have additional capacity, i.e. Chiron, have virtually no interest in small biotech companies that may have only one or two products. This leaves these smaller companies scrambling to find a place to produce material for pre-clinical and clinical testing.

Based on this information, there are very few alternatives for small companies, university investigators or government agencies to do process development, scale-up a process, and produce sufficient material for pre-clinical and clinical testing. The government does have two small cGMP facilities, the $\mathrm{NCl}$ facility at Ft. Detrick in Frederick, Maryland, and Walter Reed Army Institute for Research (WRAIR). Unfortunately, these two facilities can only handle a limited number of products. In my own experiences with DoD and the botulism vaccine program, the contractor in charge of transitioning into clinical production the process technology developed at the UN-L Biological Process Development Facility had a difficult time finding suitable contractors that were interested.

This is where universities can play a role in assisting the civilian bioterrorism program headed by NIAID, the DoD bioterrorism program, and small biotechnology companies. In addition to providing access to expensive facilities and expertise, such facilities at universities can be an excellent place to train both engineering and science students in a FDA-regulated environment.

The focus of this talk is to describe how the University of Nebraska Lincoln (UN-L), the Department of Chemical Engineering, and the UN-L Biological Process Development Facility developed a non-traditional program that blends basic and applied research with process development and scale-up and manufacturing of clinical material in a current Good Manufacturing Practice (cGMP) facility. This facility has successfully helped companies and government agencies bring biopharmaceutical molecules to clinical testing.

\section{Why Should Universities Move into this Role?}

With regard to science and engineering, a university's primary mission is to educate students and expand the basic knowledge of science and engineering for the betterment of society. Universities are also involved in applied research and technology that is of value to society. With regard to the fight against 
biological threats, universities will play a very important role in the classical sense, by understanding the basic science of different pathological organisms and toxins with the intent of identifying molecules as vaccine candidates and targets for therapeutics. Universities excel in this role.

Recently I was involved in a Blue Ribbon Panel held February 4-5, 2002 to discuss the research agenda for NIAID's bioterrorism program. Dr. Anthony Fauci, Director of NIAID, gave the opening speech to the conference and made what traditional scientific circles would call a very unusual request of NIAIDfunded scientists. Dr. Fauci told the audience that the American public and the President expect scientists working on bioterrorism to "get products into vials." This is a profound statement coming from $\mathrm{NIH}$, which prides itself on serving the very basic science needs of society. This is not to say the NIH does not support STTR and SBIR type of proposals; in fact, NIH has a very active program on commercializing potential technology. It was apparent at the meeting that scientists were asked to think outside the box in order to serve society's need to combat bioterrorism.

NIAID is asking scientists to incorporate a more company-like approach to drug discovery. NIAID still insists on good basic science on pathogenesis and mechanisms of toxin, but if a promising drug candidate comes along, that molecule needs to be "transitioned" into development. This is where academicians tend to struggle and companies succeed. Companies do not fail because they were unable to make material for clinical trials. Companies fail because the product did not live up to its clinical expectations. The BPDF's role is to operate in that realm between drug discovery and clinical trials and to assist scientists, government agencies and companies' transition molecules through Phase $1 / /$ clinical testing.

\section{The Mission of the Biological Process Development Facility at UN-L}

The BPDF has three distinct missions. The first is to educate students in bioprocessing in an FDA-mandated environment. The second is to expand and disseminate basic and applied science and engineering knowledge as it relates to all aspects of recombinant protein expression and production through publications. The third is to assist companies and government agencies in bringing biotherapeutic products to clinical trials.

\section{Description of the Biological Process Development Facility at UN-L}

The BPDF is a state-of-the-art biological process research and development facility that specializes in developing manufacturing processes for vaccines and therapeutic proteins derived from recombinant organisms. The BPDF is a "turn key" facility that deals with all aspects of process research and development including, molecular biology, fermentation and protein purification, 
analytical methods, quality control, quality assurance, process scale-up, and manufacturing of Phase I clinical material under cGMP as mandated by the FDA.

The BPDF trains students for the biotech industry, disseminates information on biological process research, and services the clinical manufacturing needs of industry, non-profit institutions, and government. The BPDF accomplishes this mission by working in an environment similar to industry on relevant projects. The BPDF will either develop processes for transfer back to the client or produce the product in our own pilot plants. This includes the production of materials that are currently being used for human clinical trials, which requires the operation under cGMP.

The BPDF is recognized as one of the leading university bioprocess facilities specializing in methylotrophic yeast, Pichia pastoris. The BPDF has 35 full-time staff and employs 6 to 10 engineering undergraduate students annually, providing them with a "real world" work experience. The BPDF has an extensive network with both small and large biotechnology companies providing potential employment opportunities, especially for students who have worked in the facility. The BPDF gives students a unique opportunity to gain practical engineering and research experience while remaining in an academic environment that allows for continued education and personal development.

The BPDF is composed of eight units: Molecular Biology Laboratory (MBL), Fermentation Development Laboratory (FDL), Purification Development Laboratory (PDL), Analytical Methods Development (AMD) and Quality Control Laboratory (QCL), Fermentation Pilot Plant (FPP), Purification Pilot Plant (PPP), and Quality Assurance (QA). These combined entities provide the BPDF with "turn key" capabilities to clone a gene of interest, develop the analytical methods and the process, and manufacture the protein under cGMP for Phase I/II clinical trials.

Molecular Biology Laboratory: The MBL conducts research in protein expression primarily in $P$. pastoris, in particular promoter regulation and strain improvement.

Fermentation Development Laboratory: The FDL conducts research in fermentation modeling, methanol control strategies, and interaction of control strategies on protein expression in $P$. pastoris. The other function of the FDL is to develop fermentation processes at the $5 \mathrm{~L}$-scale and transfer that process to the fermentation pilot plant. The FDL must take into account fermentation scaleup issues when developing the fermentation process. It is essential that the process is straightforward and generates a consistent yield with high product quality and can be readily transferred to the pilot plant $(500 \mathrm{~L})$. This is invaluable to a company that wants to make a product that will eventually be used in human clinical trials. 
Purification Development Laboratory: The PDL performs bench-scale protein purification research and develops scalable purification processes for production of proteins under cGMP. The PDL is capable of both basic protein chemistry, process development, and scaling-up a purification process into production.

Fermentation Pilot Plant: The purpose of the FPP is to scale-up a fermentation process that has been developed in the FDL, and produce cGMP material for clients. To a lesser degree the FPP conducts process research and has been involved in publishable activities. The FPP has fermentation capabilities up to $400 \mathrm{~L}$ working volume and is capable of harvesting/down stream processing using cell disruption, centrifugation, crossflow membrane filtration, or expanded bed absorption.

Purification Pilot Plant: The purpose of the PPP, which compliments the FPP in scale, is to scale-up a process that has been developed in the PDL and produce cGMP material for clients. To a lesser degree, the PPP conducts process research and has been involved in publishable activities. Both pilot plants operate under cGMP.

Analytical Methods Development: The purpose of the AMD is to conduct research in analytical methods and to develop in-process analytical methods to support process development and the pilot plants. The AMD is also responsible for developing analytical methods for testing raw materials and methods for final product release. The AMD, when required, is responsible for validating methods. Once AMD has developed a method it is then transferred to Quality Control.

Quality Control: $Q C$ is responsible for providing analytical support to the BPDF. The QC laboratory is responsible for more sophisticated assays, such as HPLC, ELISA and endotoxins. The development laboratories are responsible for routine protein assay and electrophoresis. The QC lab does all analytical methods for the pilot plants.

Quality Assurance: The function of QA is to insure the BPDF is in compliance for both Good Laboratory Practices, GLP, (FDL and PDL) and cGMP (FPP and PPP). The BPDF serves its clients by providing either a process that can be transferred into a GMP facility or production of GMP material for clinical trials. In either case, QA, in collaboration with BPDF staff, generates the necessary documents to successfully transfer/produce the product. The biggest responsibility of the QA program is to bring the BPDF into cGMP compliance.

The Research Component of the BPDF: The BPDF sustains an active research program by supporting Research Assistant Professors (RAP) in critical areas in bioprocessing. The BPDF supports RAPs in molecular biology, fermentation modeling, protein purification, and analytical methods. The BPDF supports a tenure track faculty position in quality assurance/analytical methods. Each individual is expected to have a research program and generate 
publications that further elevate the BPDF. The RAP's are involved in grant and contract writing that is either independent or multi-disciplinary within the BPDF or the university.

cGMP Capabilities: The ability of the BPDF to manufacture Phase I clinical material under cGMP is what makes the BPDF unique among university bioprocess facilities. Bringing the BPDF into CGMP compliance to produce Phase I clinical material has taken nearly 4 years, and is a process that is always evolving. A significant amount of resources (equipment, validation, establishment of systems and documentation) were needed to establish cGMP compliance. At present nearly $30 \%$ of the BPDF staff is dedicated to QA.

This capability is what attracts clients to UN-L to do bioprocess research and development. Nearly all of the BPDF clients have the same goal, which is to bring a molecule to clinical trials. Once a molecule has been discovered and preliminary studies suggest a therapeutic use, the next stage for the client is to develop a process that can be scaled-up to produce clinical material. This is not a trivial matter and includes everything from additional strain development and validation to final product tests for release of the product. Typically, it takes 9 to 12 months for the BPDF to develop a process capable of producing cGMP material. Once a process is developed it only takes 3 to 4 weeks in the pilot plant to produce a clinical lot.

It is this step between process development and clinical manufacturing, i.e. technology transfer, which can take 2 to 6 months if the process needs to be moved from one facility to another, i.e. from the facility that did process development to the cGMP production facility. If this step can be eliminated, it can save a company a significant amount of time and money. It is said that for each day a product launch is delayed it costs a company one million dollars/day in revenue. Thus, from a client's perspective one stop shopping is preferred.

Educational Aspects of the BPDF: The Department of Chemical Engineering, the departmental home of the BPDF, and the BPDF provide a very unique opportunity for science and engineering students to receive "real world" training in a cGMP environment. As a discipline, bioprocessing must be affiliated with a chemical and/or biochemical engineering program. The curriculum in chemical engineering is best suited for students in bioprocessing. It is critical that these students receive the necessary background is transport phenomena, kinetics, thermodynamics, process control, reactor design, and unit operations.

The BPDF encourages students to work in the BPDF during the school year, 10 to 15 hours per week, and to work full time through the summer. Students following this regime have succeeded very well when competing for employment and have had an opportunity to come up through the ranks in the BPDF. By the time students are juniors or seniors they are working for a scientist in a capacity similar to a graduate student. Some of the better students receive 
cGMP training and work in the pilot plants. They receive training in writing standard operating procedures and working with batch records.

The BPDF and the Army Bioterrorism Program: The BPDF has been working with USAMRIID for the last seven years on research and development of processes to produce vaccines against botulism toxin. The BPDF has transitioned five of the seven serotypes of Clostridium botulinum, A, B, C, E and $F$. Serotypes A and B have been officially transitioned to a contract research organization that is responsible for producing clinical material. This was accomplished by working with Dr. Leonard Smith, chief scientist at USAMRIID, who was responsible for discovering vaccine candidates. Dr. Smith worked closely with the BPDF to develop processes for transitioning the vaccine candidates into clinical trials. The BPDF is funded to work with USAMRIID an additional three years on additional vaccines and therapeutics, i.e. MAbs. The BPDF is involved in all aspects of process development from strain development to process scale-up for USAMRIID. Presently, work for the Army comprises about $20-25 \%$ of the BPDF total activity.

The Civilian Bioterrorism Program and University Involvement: For the civilian bioterrorism program to succeed it will be critical for NIAID-funded scientists doing drug discovery and pathogenesis research to initiate a collaborative relationship as soon as possible with a process scale-up group. There are many things that can be done early on in drug discovery that will save the process development scientist a significant amount of time and effort. The BPDF works very closely with USAMRIID at all stages of drug discovery.

Major universities typically have the biological scientists (drug discovery) and the biochemical engineers (process development and scale-up) that are necessary to accelerate the transition from discovery to a vial. The challenge is getting the two parties collaborating and making decisions based on what is required by the FDA. This is not the typical thought process for a university scientist, but it is necessary to accelerate the transition of product candidates into vials.

BPDF Funding: The BPDF is entirely funded by grants and contracts, except for the Director's salary. The annual contract and grant activity is from $\$ 3$ to $\$ 4$ million per year.

Future Direction of the BPDF: The BPDF is currently in the Food Processing Center on East Campus at the University of Nebraska - Lincoln. In April - May of 2003 , the BPDF and two biochemical engineering faculty will occupy $10,000 \mathrm{ft}^{2}$ of bioprocess research laboratories and $2,500 \mathrm{ft}^{2}$ offices dedicated completely to bioprocessing and bioengineering research on the third floor of the new Othmer Hall. In addition, there will be $3,000 \mathrm{ft}^{2}$ of non-GMP research pilot plants in the basement. Also in the plans is a new $10,000 \mathrm{ft}^{2} \mathrm{cGMP}$ pilot plant with half of the 
facility dedicated to yeast/bacteria and the other half to mammalian cell culture. Maximum reactor size in each facility will be 1,000 L.

Future Direction of Bioterrorism Research and Development at Universities

I would recommend that universities form bioterrorism research clusters. This is initiated by identifying a particular area of research, such as a pathogen or diagnostics, as an emphasis. In the case of vaccine or therapeutic discovery, it will be important to identify all of the necessary resources and present a plan that takes into consideration all aspects of drug discovery and clinical manufacturing. Taking this approach will insure that the scientific community will have the quickest response in bringing a product to a vial. 